\title{
Effectiveness of the Preventive Intervention of Chromosomal Disorders of Iran's Community Genetics Program: Application of Bayesian Network
}

\author{
Seyyedeh Sara Azimi ${ }^{1,2}$, Sama Ashraf Samavat ${ }^{2}$, Vahid Rezaei Tabar $^{3,4}$, Hamid Soori ${ }^{1,5 *(1)}$ \\ Received: 7 Mar 2021 \\ Published: 11 Oct 2021
}

\begin{abstract}
Background: Evidence-based policymaking for the genetic preventive interventions at the community level requires information on the effectiveness of interventions in the operational areas taking into account the characteristics of health system and customer behaviour. These information are limited in many low- and middle-income countries. In this study, we estimated the effectiveness of preventive interventions for chromosomal disorders using the conceptual framework of Iran's community genetics program (ICGP) using a Bayesian Network as a modeling method in limited access situation to the complete and accurate observational data.

Methods: Expert elicitation method based on global and national scientific evidences was applied to determine the structure of the Bayesian Network (BN) and to quantify the probability of nodes. The nomological and face validity of the network was checked. Also, a sensitivity analysis against the sources of uncertainty of probabilities was conducted.

Results: By ICGP interventions, 63\% (95\% CI, 0.55-0.71) of all chromosomal disorders can be prevented, which is responsible for $80 \%$ (95\% CI, 0.76-0.84) and 38\% (95\% CI, 0.31-0.45) reduction of expected baseline birth prevalence of trisomis and other autosomal disorders, respectively. Improving the access to and the uptake of screening service can also result in a $12 \%$ and $11 \%$ increase in effectiveness, respectively.

Conclusion: Effectiveness of ICGP's intervention is between the same interventions' effectiveness in Western Europe and the Eastern Mediterranean region. Opportunities for increasing the uptake of and the access to the interventions are strengthening the public genetic literacy and implantation of a system of laboratory sample transfer at the side of the utilization of telehealth for delivering the counseling services at remote areas.
\end{abstract}

Keywords: Bayesian Network, Effectiveness, Preventive Intervention, Congenital Disorder, Chromosomal Disorders, Iran's Community Genetics Program

Conflicts of Interest: None declared

Funding: None

*This work has been published under CC BY-NC-SA 1.0 license.

Copyright $\bigcirc$ Iran University of Medical Sciences

Cite this article as: Azimi SS, Samavat SA, Rezaei Tabar V, Soori H. Effectiveness of the Preventive Intervention of Chromosomal Disorders of Iran's Community Genetics Program: Application of Bayesian Network. Med J Islam Repub Iran. 2021 (11 Oct);35:132. https://doi.org/10.47176/mjiri.35.132

\section{Introduction}

Genetic and congenital disorders are a collection of het-

erogeneous disorders needing different interventions to be

\section{Corresponding author: Dr Hamid Soori, hsoori@sbmu.ac.ir}

Department of Epidemiology, School of Public Health and Safety, Shahid Beheshti University of Medical Sciences, Tehran, Iran

2. Genetics Office, Noncommunicable Disease Management Centre, Ministry of Health and Medical Education, Tehran, Iran

3. Department of Statistics, Allameh Tabataba'i University, Tehran, Iran

4. School of Biological Sciences, Institute for Research in Fundamental Sciences (IPM),Tehran, Iran

5. Safety Promotion and Injury Prevention Research Center, Shahid Beheshti University of Medical Sciences, Tehran, Iran $\uparrow$ What is "already known" in this topic:

In previous studies, the effectiveness of preventive interventions was calculated using the interventions on groups of congenital disorders at the level of the World Health Organization regions, without assessing the impact of the differences in the uptake and access level.

$\rightarrow$ What this article adds:

Decomposition of the effectiveness to its components at the 3 levels of intrinsic characteristics of the intervention, behavioral characteristics of target groups, and characteristics of the health system's services delivery was conducted in this study for the first time. Moreover, the Bayesian network can also be applied in the communities interested in implementing genetic programs at the community level if domestic probabilities are considered in the model. 
controlled or prevented. Improving children's health indicators affected by genetic and congenital disorders was impeded because of a lack of proper prioritization of the interventions in many co untries (1). Iran is one of the pioneer countries in implementing community-based genetic programs $(2,3)$. The model was developed into a comprehensive program of community-based control and prevention of genetic and congenital disorders, which was integrated into Iran's health system over the last 2 decades $(2,4,5)$. In Iran's community genetics program (ICGP), interventions to prevent affected birth of genetic and congenital disorders are designed based on the nature and epidemiological characteristics of disorders on the one hand, and feasible intervention strategies on the other. However, assessing the effectiveness of applied intervention and estimating the effectiveness of further planed interventions is necessary for improving the evidencebased priority setting about this complex interventions.

Determining the effectiveness of health interventions is usually done by conducting the primary studies on observational data or secondary study conducting on results of the existing primary studies $(6,7)$. In the context of complex interventions, the simple application of systematic review and meta-analysis studies are not a sufficient approach because of the multidimensional aspects of complex interventions, including multiplicity of interacting components, groups and organizational levels targeted by the interventions, variety in the outcomes, and degree of flexibility or compatibility to the area of implementation (8). In such cases, a tool is needed to integrate the existing scientific evidence and experts' opinions on the structure and strength of the relationship between the variables determining the effectiveness. The Bayesian networks (BNs) were introduced as an effective tool to estimate the effectiveness of complex health interventions (9).

BNs are a tool for visualizing the relationships among variables in a complex network of causal or noncausal relationships and quantifying these variables' conditional probabilities relative to each other. Therefore, conducting a BN consists of 2 aspects: "structure learning" and "parameter learning". Both aspects of BNs can be constructed using the machine learning algorithms from a set of observational data or using a combination of existing pieces of knowledge from scientific evidences or expert opinions, which in the second case, $\mathrm{BN}$ is called a Belief $\mathrm{BN}$ (10).

BNs also allow the sensitivity analysis for all aspects of the network that provide the opportunity to investigate the parameters influencing the overall effectiveness of the intervention as well as the value of information and decision analysis regarding the alternative interventions. The possibility of investigating the effectiveness of scenarios in the desired intervention that have not been implemented yet or are proposed as the alternatives is one of the BN's unique features as a modeling tool $(9,11)$. Thus far, BN has been used in several studies as a tool to analyze the networks of various health outcomes in the field of communicable, noncommunicable diseases, public health, cognitive and behavioral sciences, et cetera. Investigating the effect of interventions on the outcomes using BNs is an area that has received more attention over recent years $(9,12)$. Furthermore, in this study, interventions' effectiveness to prevent chromosomal disorder in Iran was estimated as an example of interventions for preventing genetic and congenital disorders because of the framework of Iran's community genetics program.

\section{Methods}

\section{Study Design}

This was a secondary study based on a modeling method combining the best available data on different acpects of effectiveness of the ICGP according to the national reports when available and expert opinion in limitation of abservational data. Details of modeling and data collection methods are described below.

\section{Determining BN Structure}

To determine the structure of BN using the conceptual framework of the ICGP, the target genetic disorder of the program was divided into 3 main categories of chromosomal disorders, abnormalities, and inherited disorders, including single-gene disorders and diseases with genetic risk factors, extracted by grouping criteria introduced in the Modell Global Database study (MGDb) (1). For sex chromosomal disorders, pregnancy termination for Turner and Klinefelter syndromes are not legally allowed, except for the mosaicism cases that lead to hermaphroditism or concomitation with major abnormalities. Therefore, sex chromosomal disorders were not assumed as preventable chromosomal disorders in this study.

Each preventive subprogram of ICGP includes 2 aspects of intervention: first, identifying at risk cases (including specific screening and/or opportunistic genetic risk assessment), and second, the prevention of affected birth (PAB). Genetic counselling, genetic diagnosis services, and genetic literacy education are supplementary parts of each preventive subprogram. The identification strategy, depending on the disorder's nature and its relative prevalence, is either done through a specific screening or opportunistic risk identification program. Opportunistic risk identification pathways through marriage questionnaire and integrated in routine health services, seeking to identify a history of genetic disease with a diagnosis or recurrence of the unknown suspected genetic diseases in the family is a strategy to identify rare genetic diseases. PAB is the active pursuance of women at risk of having children with genetic disorders, which were identified through 2 strategies mentioned above. PAB leads to timely risk identification of pregnancies, genetic testing of fetus, and counseling for termination of the pregnancy of affected fetus by the mother's informed decisions. The conceptual framework of the interventions of ICGP are shown in Figure 1 .

The effectiveness of each subprogram is affected by 2 aspects of risk identification and prevention of affected birth, which are mentioned above in 3 dimensions of efficacy, access, and uptake of these 2 interventions. Efficacy as each intervention's intrinsic trait is defined as a success rate (detection rate for identification intervention and prevented affected birth rate for PAB in the ideal conditions). 


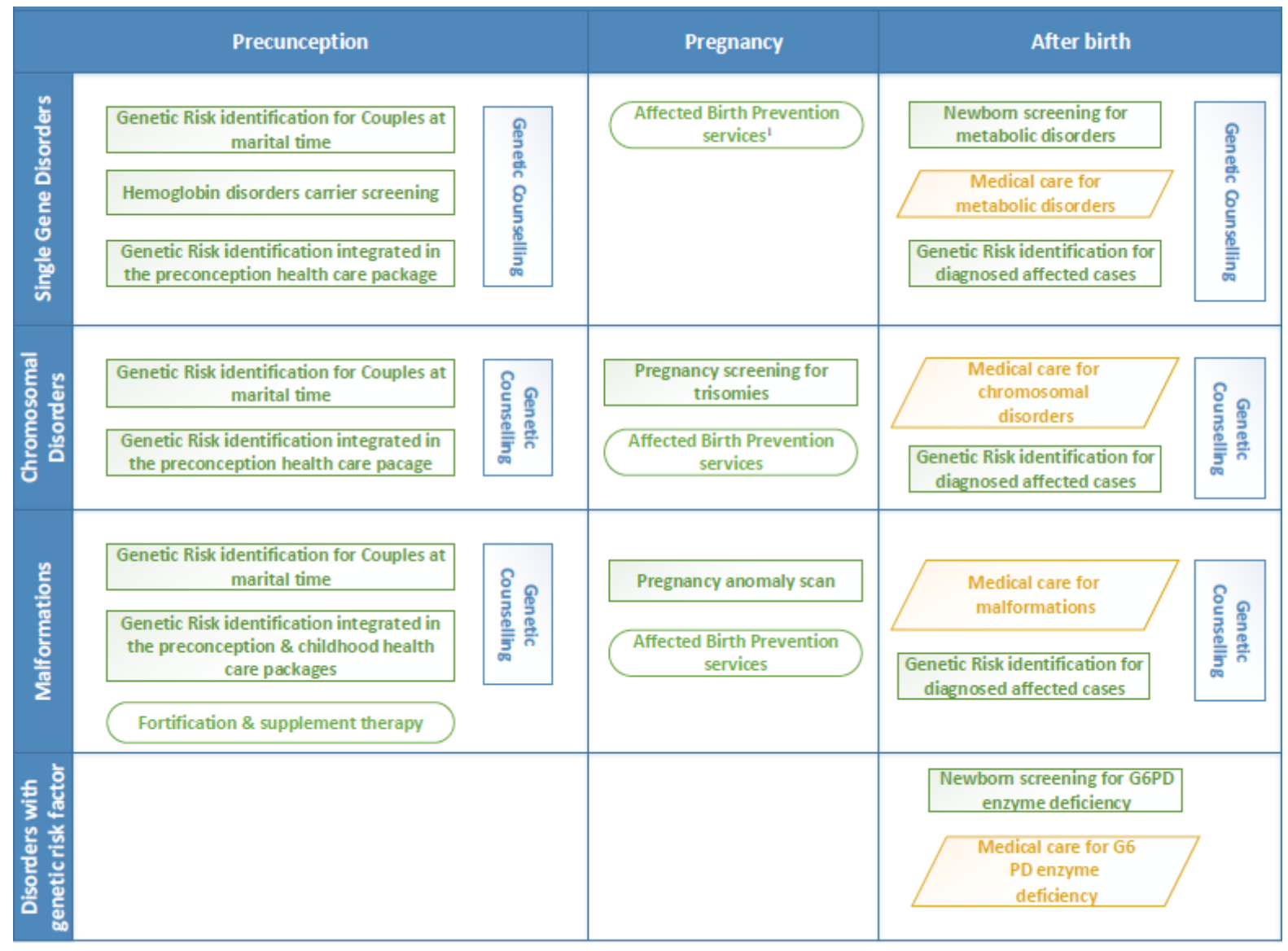

${ }^{1}$ Termination of pregnancy in the case of mothers' request after genetic testing for fetus and posttest genetic counselling

Fig. 1. Conceptual Framework of Preventive Interventions of Community Genetics Program of Iran

However, access and uptake are traits of the implementation condition of intervention that can modify interventions' intrinsic efficacy.

The BN structure generally consists of 2 categories of variables: the parent node and the child node. Each child node can itself be the parent of other nodes in the network. Any node, which is just a parent and not a child of another node, is called a root node. The set of parents and child nodes is conditionally independent of all the other nodes, which given the specific node, it is named Markov blanket of that node. Based on the Bayesian probability theory, essential assumptions for a Markov chain is that a node must be conditionally independent of the entire network, given its Markov blanket. In other words, a Markov network is a direct acyclic graph without any return pathway to the starting point (12). The 3 aspects of efficacy, access, and uptake of each of the 2 interventions of risk identification and $\mathrm{PAB}$ of each of preventive subprograms, used as parent nodes, are important for the child node of the intervention's effectiveness in $\mathrm{BN}$.

\section{Determining the Probability Distribution of the Parent Nodes of $B N$}

To quantify the probability distributions of root and parent nodes, we reviewed the existing relevant scientific evidence at the global and national levels (13-17). Then, the probability elicitation method based on the expert opinion was conducted to harmonize the evidence by considering the acceptable national range of the above indicators (18-21). The truncated normal distribution phase been taking into account as the prior distribution for all parent nodes, except for the distribution of PAB's efficacy. The sources of information used in expert elicitation to determine the parameters of effectiveness of prior distribution of parent nodes are presented in Table 1.

The efficacy of the questioner-based genetic risk identification has been determined based on the program's marital genetic risk identification observational data. In terms of time direction of risk identification strategies, there are 2 general categories of interventions to prevent congenital disorders: (1) prospective risk identification (PRI) in which the risk identification is performed before having the first affected child, leading to $100 \%$ prevention of affected birth theoretically; (B) retrospective risk identification (RRI) in which the risk identification for couples is performed after the birth of the first affected child with different efficacies associated with total fertility rate (TFR) and with the goal of healthy childbearing of at-risk couples (13). By assuming 2 healthy children for each atrisk couples according to the observational data from the thalassemia prevention program, we quantified efficacy distribution of the PAB using the quantitative values in- 


\begin{tabular}{|c|c|c|}
\hline Root Node & & Source of Information Used in Expert Elicitation \\
\hline Total fertility rate & & Statistical yearbook of the statistical centre of Iran (2018) (22) \\
\hline \multicolumn{3}{|l|}{ Percentage of urban resistance } \\
\hline \multirow[t]{3}{*}{ Trisomy screening } & Efficacy & $\begin{array}{l}\text { Result of methaanalysis of cost-effectivness of prenatal screening methods, } \\
\text { Walker et al (23) }\end{array}$ \\
\hline & $\begin{array}{l}\text { Access } \\
\text { Uptake }\end{array}$ & $\begin{array}{l}\text { Result of a survey of ultrasound use in prenatal health care in Iran, Sangestani et } \\
\text { al (24) }\end{array}$ \\
\hline & & And information from national electronic primary healthcare record system (25) \\
\hline \multirow[t]{2}{*}{ Opportunistic risk identification } & Efficacy & $\begin{array}{l}\text { Report of national program of marital genetic risk identification (unpublished } \\
\text { document of genetics office of of Iran's ministry of health) }\end{array}$ \\
\hline & Access & Result of Iranian healthcare utilization household survey, Vahedi et al (17) \\
\hline \multirow[t]{3}{*}{ Prevention of affected birth (PAB) } & Efficacy & $\begin{array}{l}\text { Result of effect estimation of preventive interventions for congenital disorders, } \\
\text { Blencowe et al (13) }\end{array}$ \\
\hline & Access & observational information of genetic prevention programs implemented in the \\
\hline & Uptake & $\begin{array}{l}\text { country (eg, beta-thalassemia major prevention program and phenylketonuria } \\
\text { prevention and control program) }(26,27)\end{array}$ \\
\hline
\end{tabular}

troduced in the MGDb study for PRI and RRI using a castom PDF distribution (13).

\section{Estimating the Joint probability of Effectiveness}

The joint conditional probability for each child nodes factorized as a product of several conditional distributions of parent nodes in its Markov blanket, as mentioned in equation 1 is as follows (28):

$$
P\left(X_{1}, \ldots, X_{2}\right)=\prod_{I=1}^{n} P\left(X_{i} \mid P_{a}\left(X_{i}\right)\right)
$$

$P\left(X_{i} \mid P_{a}\left(X_{i}\right)\right)$ denotes the conditional probability of each node given its parents.

In the present study, because of the simultaneous presence of continuous and discrete variables in the network, the hybrid forward sampling algorithm was used to generate 10,000 samples to generate the posterior probability distribution of nodes. The mean and SD of the posterior distribution of effectiveness nodes have been used to calculate this index's 95\% CI. BN design and probability calculation are performed using GeNIe Software Academic Version 2.3.

\section{Validation of Network and Sensitivity Analysis}

In a broader sense, validity is defined as "describing the system that is intended to describe both the output and the mechanism by which the output is generated." Furthermore, in the case of belief BNs, the structure of the network is based on the researchers' opinion and not machine learning algorithms, and different methods are used to check the validity of the model compared with the quanti- tative validity check methods in data-based modeling. In the present study, the validity of model structure in terms of nomological validity and face validity was investigated by examining the compatibility of model structure with other existing models and experts' opinion (29). Also, 1way sensitivity analysis was conducted based on changing each important parent's probability of effectiveness in the spectrum of acceptable values based on experts' opinions.

\section{Ethics Statement}

The study was approved by Shahid Beheshti University of Medical Sciences Ethics Committee (No.IR.SBMU.PHNS.REC.1398.044).

\section{Results}

The $\mathrm{BN}$ of prevention interventions of chromosomal disorders is structured by 4 type of parent nodes that determine the effectiveness nodes, as shown in Figure 2.

The effectiveness of chromosomal disorders prevention interventions with 2 strategies, "pregnancy screening for trisomies" and "genetic risk identification for other autosomal disorders," based on their parent nodes of efficacy, acceptance, and access is listed in Table 2.

In total, $63 \%$ (95\% CI, 0.55-0.71) of all chromosomal disorders can be prevented by ICGP interventions, which are responsible for $80 \%$ (95\% CI, 0.76-0.84) and $38 \%$ (95\% CI, 0.31-0.45) reduction of expected birth prevalence of trisomis and other autosomal disorders, respectively.

The sensitivity analysis results of parent nodes of 2 effectiveness nodes in the network is shown in Figure 3.

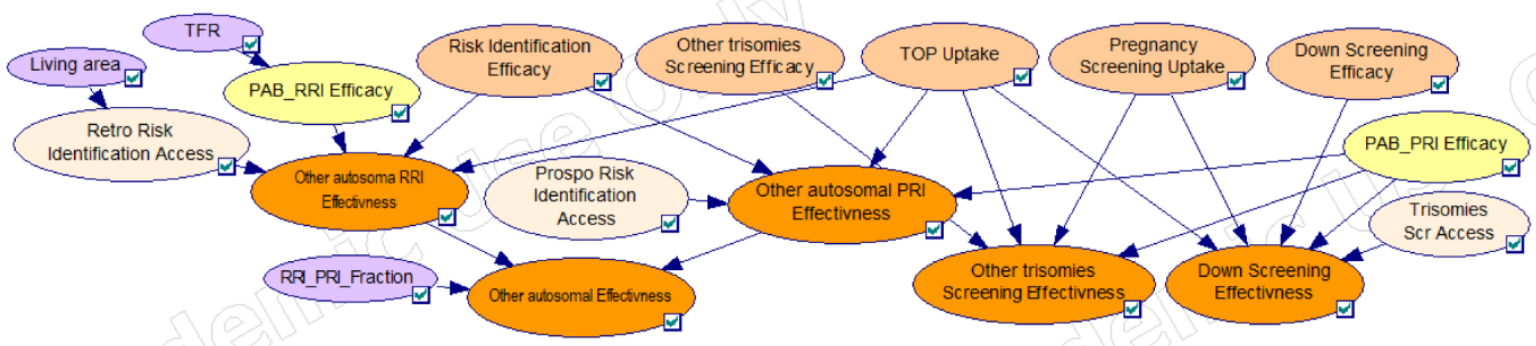

Fig. 2. The Bayesian Network of Prevention Interventions of Chromosomal Disorders 


\begin{tabular}{|c|c|c|c|c|}
\hline Disorder Category & $\begin{array}{c}\text { Aspect of } \\
\text { Intervention }\end{array}$ & $\begin{array}{c}\text { Components of } \\
\text { Intervention }\end{array}$ & Subcategory & Percentage $(95 \% \mathrm{CI})^{1}$ \\
\hline \multirow[t]{8}{*}{ Trisomies } & Efficacy & Pregnancy screening & Trisomy 21 & $0.93(0.91-0.95)$ \\
\hline & & & Trisomy $13 \& 18$ & $0.95(0.93-0.97))$ \\
\hline & & $\mathrm{PAB}^{2}$ & $\mathrm{PRI}^{3}$ & $0.99(0.98-1)$ \\
\hline & Access & & & $0.90(0.96-0.94)$ \\
\hline & Uptake & Screening & & $0.97(0.95-0.99)$ \\
\hline & & $\mathrm{TOP}^{4}$ & & $0.98(0.97-0.99)$ \\
\hline & Effectiveness & Trisomy 21 & & $0.80(0.76-0.84)$ \\
\hline & & Trisomy $13 \& 18$ & & $0.90(0.87-0.93)$ \\
\hline \multirow[t]{10}{*}{ Other autosomal } & Efficacy & $\begin{array}{l}\text { Questionnaire based } \\
\text { genetic risk identifica- } \\
\text { tion }\end{array}$ & & $0.70(0.65-0.75)$ \\
\hline & & PAB & PRI & $0.99(0.98-1)$ \\
\hline & & & $\mathrm{RRI}^{5}$ & $0.15(0.12-0.17)$ \\
\hline & Access & & PRI & $0.99(0.98-1)$ \\
\hline & & & RRI & $0.85(0.78-0.92)$ \\
\hline & Uptake & TOP & & $0.98(0.97-0.99)$ \\
\hline & Effectiveness & & PRI & $0.68(0.63-0.73)$ \\
\hline & & & RRI & $0.09(0.06-0.12)$ \\
\hline & & & $\begin{array}{c}\text { Weighted mean }{ }^{6} \text { of both ap- } \\
\text { proaches }\end{array}$ & $0.38(0.31-0.45)$ \\
\hline & & & Total & $0.63(0.55-0.71)$ \\
\hline
\end{tabular}

\footnotetext{
${ }^{1}$ Based on the mean and SD of normal distribution as the posterior distribution of each node in the Bayesian network.

${ }^{2}$ Prevention of affected birth services, including genetic testing for fetus and posttest genetic counselling.

${ }^{3}$ Prospective risk identification strategy (risk identification for at-risk couples at marital time until preconception)

${ }^{4}$ Termination of pregnancy by mothers' request.

${ }^{5}$ Retrospective risk identification strategy (risk identification for at-risk couples after the first affected birth)

${ }^{6}$ By assuming the equal fraction for PRI and RRI.
}

A
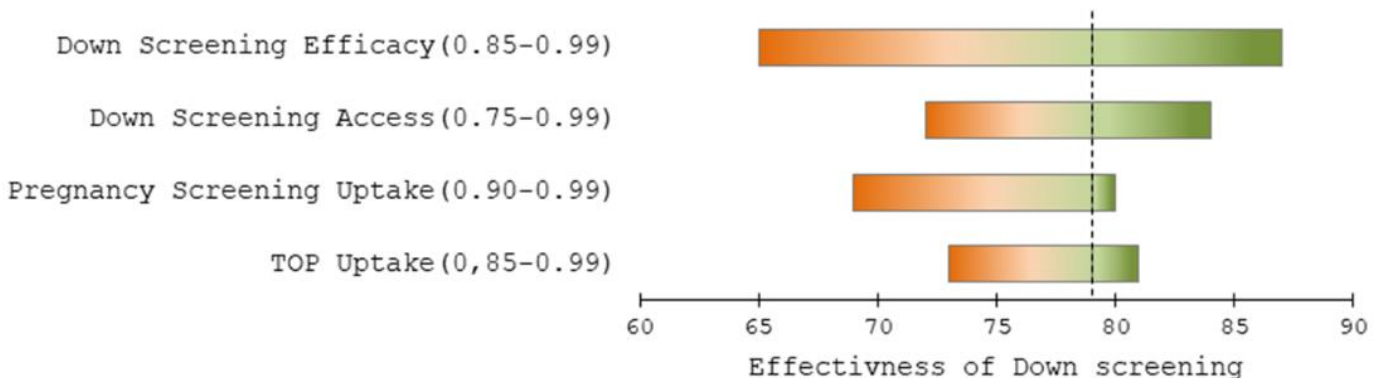

B

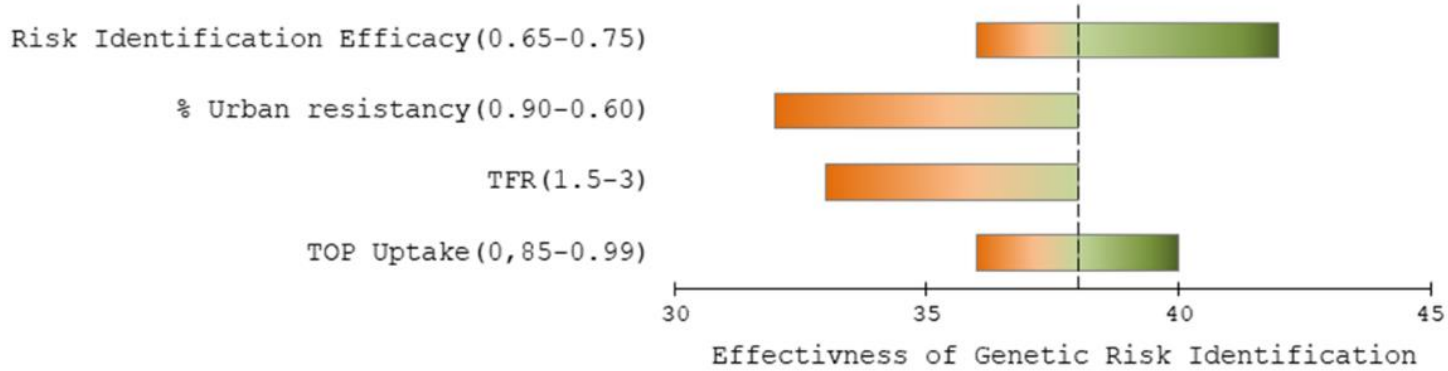

Fig. 3. Tornado Diagram of 1-Way Sensitivity analysis (A) for Down Syndrome Screening and (B) for Risk Identification of Other Autosomal and Sex chromosomal Disorders.

Sensitivity analysis results showed that the change in the quantitative amount of probability distribution of down syndrome screening effectiveness and Down screening access has the greatest effect on estimating the joint probability of effectiveness of pregnancy screening intervention for trisomy disorders. And in the order of changing the probability of these parent nodes from the minimum to the maximum acceptable value, the effectiveness of Down screening varied $22 \%$ and $12 \%$, respectively (Fig. 3-A).

Regarding the effectiveness of genetic risk identification, the efficacy of the questioner was the most important parent node, which can change the probability of its child node from $36 \%$ to $44 \%$, considering its $10 \%$ variation. In the following manner, the percent of residency in urban 
areas, as a root node of access to PHC services, and TFR, as a root node of retrospective PAB efficacy, changed the effectiveness of genetic risk identification $6 \%$ and $5 \%$, top to the ceiling of $38 \%$ as a mean of the joint probability of genetic risk identification effectiveness. Raising up to the uptake of $99 \%$ increased the mean of effectiveness to $40 \%$ (Fig. 3-B).

\section{Discussion}

The effectiveness of trisomies screening as the strategy for preventing the common genetic disorders in ICGP and the effectiveness of genetic risk identification as the strategy of preventing the relatively rare genetic disorders are estimated. Effectiveness of screening for trisomies at pregnancy as the main preventive strategy for chromosomal disorders was estimated $80 \%$ (95\% CI, 76\%-84\%) for Down syndrome and $90 \%$ (95\% CI, 87\%-93\%) for trisomy 13 and 18, which is in the range of the result of observational studies conducted in different countries (30). In total, all preventive interventions of ICGP can result in a $31 \%$ decrease in birth prevalence of chromosomal disorders in Iran, which is equal to the reduction of $63 \%$ of preventable chromosomal disorders. This reduction includes trisomies and other autosomal disorders, except for sex chromosomal disorders.

Based on the MGDb study results, as a comprehensive review of existing evidences in the congenital disorder prevention topic, effectiveness of the ICGP's itervention is lower than the effectiveness of preventive interventions for chromosomal disorders in Western Europe, which is estimated to be 0.47 (31). This difference can be the result of more effective screening strategies and higher access in high-income countries. On the other hand, compared with the effectiveness of related interventions in the East Meditation Region (EMR), which is estimated about $26 \%$, the effectiveness of preventive interventions for chromosomal disorders in Iran is higher. This difference can be explained by religious and legal permission of TOP in the case of mother requests in Iran, which is not the case in many countries in EMR (13). Besides, conducting an active and national community-based preventive program, ICGP, has an important role in this increase.

Based on the results of 1-way sensitivity analysis, the highest impact on effectiveness was attributed to Down syndrome screening efficacy that varied from $85 \%$ in the minimal scenario (first trimester combined test) to $99 \%$ in the maximal scenario (universal noninvasive prenatal testing [NIPT]), resulting in $22 \%$ change in pregnancy screening effectiveness. Choosing the optimal screening strategy needs the complete health economic analysis rather than an effectiveness study. However, our results quantified the amount of improvement of pregnancy screening effectiveness by advancing to application of more precise and accurate screening methods, such as NIPT screening strategies. Other important nodes for determining pregnancy screening effectiveness were access to and uptake of the services for Down screening by $12 \%$ and $11 \%$ change of effectiveness, respectively. Changes in the percentage of TOP uptake had the lowest effect on the effectiveness as a result of the narrower range of variability of this node.
According to this findings, by fixing the efficacy of trisomy screening at the optimal level of a national strategy (using contingent NIPT), the best opportunities for improving the effectiveness of this screening are increasing the uptake of screening by improving public genetic literacy through the operation of educational programs, improving the access of remote areas by implantation of a system of laboratory sample transfer, and utilization of telehealth for delivering the counseling services. In addition, the development of insurance coverage for costly parts of the screening progress can increase less privileged consumers' uptake. Based on the sensitivity analysis results of the effectiveness of prevention of affected birth (PAB), by assuming the equal fraction for each risk identification approach (PRI and RRI), effectiveness of genetic risk identification is $38 \%$ for preventing nontrisomic chromosomal disorders. In populations with TFR lower than 2 as what Iran is expecting in recent years, only $0.5 \%$ of interventional nontrisomic disorders can be prevented by retrospective risk identification. Despite this, PRI isolatedly has an effectiveness equal to $68 \%$, if this strategy can identify all at-risk couples before having the first affected child. Thus, emphasizing genetic self-care to increase the couples' timely attendance at the early reproductive age to the genetic counseling sections can improve PRI's effectiveness. Hence, in general, genetic risk identification does not have high effectiveness for preventing chromosomal disorders, as this intervention can approach only $5 \%$ of all preventable fraction of chromosomal disorders.

The active partnership of managers of community genetics program in the design and conducting the study led to significant advantages in the field of model validity. First, access to a strong team of experts and first-hand evidence of observational data from the implementation of health programs in the country, including published and unpublished reports, and data in relevant registration systems. Second, a correct understanding of the executive mechanisms of the community genetics program and its practical adaptation to the model of the health interventions and the results of previous studies to design the network structure. Introduced models can also be applied in the communities interested in implementing genetic programs at the community level if domestic probabilities are considered in the model.

We used the access of the target group as a proxy of utility in the network, as in most regions of Iran, the access to the active genetic services can be assumed equal to the utility of consumers, especially for the services that are necessary for legal registration of marriage. In some areas of the country, the assumption can not be achieved because of different cultural and religious beliefs and might result in a slight overestimation of the effectiveness, if we are to generalize the findings at the provincial level.

\section{Conclusion}

The effectiveness of interventions to prevent genetic disorders is affected by 3 areas of efficacy, uptake of interventions, and the percentage of access in 2 aspects of "identification" and "prevention" of each intervention. 
Despite the limited opportunity to increase each specific intervention's efficacy, the intervention's effectiveness can be improved by modifying the setting and implementation factors of the intervention, such as applying flexible service delivery approaches and beneficial informing of the target group. Besides, the Bayesian belief network was applied as a useful tool for visualizing the structure of the conceptual framework of preventive interventions of the community genetics program of Iran and for facilitating the integration of the best available international and national evidence in a scientific manner for assessing the effectiveness of complex health interventions and providing higher levels of national evidence to support evidencebased policymaking, even in the face of limited access to individual observational data.

\section{Acknowledgment}

This study was a part of a $\mathrm{PhD}$ project that was conducted with cooperation of Genetics Office of the Ministry of Health and Medical Education of Iran.

\section{Conflict of Interests}

The authors declare that they have no competing interests.

\section{References}

1. Moorthie S, Blencowe H, Darlison WM, Lawn JE, Mastroiacovo P, Morris JK, et al. An overview of concepts and approaches used in estimating the burden of congenital disorders globally. J Community Genet. 2018;9(4):347-62.

2. Aghajani H, Samavat A, Haghazali M, Valizadeh F, Sarbazi G. Primary Health Care: An Approach to Community Control of Genetic and Congenital Disorders. Iran J Public Health. 2009;38(Supple 1).

3. Behnam B, Zakeri M. Genetics and genomic medicine in Iran. Mol Genet Genomic Med. 2019;7(2):e00606.

4. Atri Barzanjeh S, Behshid M, Hosseini MB, Ezari M, Taghizadeh M, Dastgiri S. Community Genetic Services in Iran. Genet Res Int. 2012;2012:129575.

5. Christianson A, Streetly A, Darr A. Lessons from thalassaemia screening in Iran. BMJ. 2004;329(7475):1115-7.

6. Minary L, Trompette J, Kivits J, Cambon L, Tarquinio C, Alla F. Which design to evaluate complex interventions? Toward a methodological framework through a systematic review. BMC Med Res Methodol. 2019;19(1):92.

7. Skivington K, Matthews L, Craig P, Simpson S, Moore L. Developing and evaluating complex interventions: updating Medical Research Council guidance to take account of new methodological and theoretical approaches. Lancet. 2018;392:S2.

8. Möhler R, Köpke S, Meyer G. Criteria for Reporting the Development and Evaluation of Complex Interventions in healthcare: revised guideline (CReDECI 2). Trials. 2015;16:204.

9. Stewart GB, Mengersen K, Meader N. Potential uses of Bayesian networks as tools for synthesis of systematic reviews of complex interventions. Res Synth Methods. 2014;5(1):1-12.

10. Kyrimi E, McLachlan S, Dube K, Neves MR, Fahmi A, Fenton N. A Comprehensive Scoping Review of Bayesian Networks in Healthcare: Past, Present and Future. arXiv:200208627. 2020.

11. Ades AE, Sculpher M, Sutton A, Abrams K, Cooper N, Welton N, et al. Bayesian methods for evidence synthesis in cost-effectiveness analysis. Pharmaco Economics. 2006;24(1):1-19.

12. McLachlan S, Dube K, Hitman GA, Fenton NE, Kyrimi E. Bayesian networks in healthcare: Distribution by medical condition. Artif Intell Med. 2020;107:101912.

13. Blencowe H, Moorthie S, Darlison MW, Gibbons S, Modell B, Bittles $\mathrm{AH}$, et al. Methods to estimate access to care and the effect of interventions on the outcomes of congenital disorders. J Community Genet. 2018.

14. Fullman N, Yearwood J, Abay SM, Abbafati C, Abd-Allah F,
Abdela J, et al. Measuring performance on the Healthcare Access and Quality Index for 195 countries and territories and selected subnational locations: a systematic analysis from the Global Burden of Disease Study 2016. Lancet. 2018;391(10136):2236-71.

15. Haghi M, Rajabi G. Health Care Services Utilization in Iran. Iran J Public Health. 2017;46(6):863-4

16. Anjomshoa M, Mousavi SM. Regional disparities in the distribution of health care facilities: building evidence for evidence-based policy making. Iran J Public Health. 2014;43(7).

17. Vahedi S, Rezapour A, Mohammadbeigi A, Khosravi A. Economic Inequality in Outpatient Healthcare Utilization: The Case of Iran. J Res Health Sci. 2018;18(3):e00424.

18. Soares MO, Sharples L, Morton A, Claxton K, Bojke L. Experiences of Structured Elicitation for Model-Based Cost-Effectiveness Analyses. Value Health. 2018;21(6):715-23.

19. O’Hagan A. Expert Knowledge Elicitation: Subjective but Scientific. Am Stat. 2019;73(sup1):69-81.

20. Johnson SR, Tomlinson GA, Hawker GA, Granton JT, Feldman BM. Methods to elicit beliefs for Bayesian priors: a systematic review. J Clin Epidemiol. 2010;63(4):355-69.

21. Butler AJ, Thomas MK, Pintar KD. Systematic review of expert elicitation methods as a tool for source attribution of enteric illness. Foodborne Pathog Dis. 2015;12(5):367-82.

22. Statistical Center of Iran, Statistical yearbook of the country. 2018:3177.

23. Walker BS, Nelson RE, Jackson BR, Grenache DG, Ashwood ER, Schmidt RL. A Cost-Effectiveness Analysis of First Trimester NonInvasive Prenatal Screening for Fetal Trisomies in the United States. PLoS One. 2015;10(7):e0131402-e.

24. urvey of prenatal screening policies in Europe for structural malformations and chromosome anomalies, and their impact on detection and termination rates for neural tube defects and Down's syndrome. BJOG. 2008;115(6):689-96.

25. Integrated Health Record System of Iran Tehran2020 [Available from: http://dapa.ir/en/2018/09/05/sib-integrated-health-recordsystem/.

26. Hashemieh $M$, Timori Naghadeh $H$, Tabrizi Namini $M$, Neamatzadeh H, Hadipour Dehshal M. The Iran Thalassemia Prevention Program: Success or Failure? Iran J Ped Hematol Oncol. 2015;5(3):161-6.

27. Shokri M, Karimi P, Zamanifar H, Kazemi F, Badfar G, Azami M. Phenylketonuria screening in Iranian newborns: a systematic review and meta-analysis. BMC Pediatr. 2020;20(1):352.

28. Kruijshaar ME, Barendregt JJ, Hoeymans N. The use of models in the estimation of disease epidemiology. Bull World Health Organ. 2002;80(8):622-8.

29. Pitchforth J, Mengersen K. A Proposed Validation Framework for Expert Elicited Bayesian Networks. Expert Syst Appl. 2013;40(1):162-7.

30. Yao Y, Liao Y, Han M, Li SL, Luo J, Zhang B. Two kinds of common prenatal screening tests for Down's syndrome: a systematic review and meta-analysis. Sci Rep. 2016;6:18866-

31. Moorthie S, Blencowe H, Darlison MW, Gibbons S, Lawn JE, Mastroiacovo $\mathrm{P}$, et al. Chromosomal disorders: estimating baseline birth prevalence and pregnancy outcomes worldwide. J Community Genet. 2017. 